\title{
Investigation of Laminar Separation Bubble on Flat Plate with Adverse Pressure Gradient: Time-Averaged Flow Field Analysis
}

\author{
Yonglei Qu, ${ }^{1}$ Dario Barsi, ${ }^{2}$ Daniele Simoni, ${ }^{2}$ Pietro Zunino, ${ }^{2}$ and Yigang Luan $\mathbb{D}^{1}$ \\ ${ }^{1}$ College of Power and Energy Engineering, Harbin Engineering University, Harbin, China \\ ${ }^{2}$ Department of Mechanical, Energy, Management and Transport Engineering, University of Genova, Genova, Italy
}

Correspondence should be addressed to Yigang Luan; yigangluan@hrbeu.edu.cn

Received 22 November 2020; Revised 13 January 2021; Accepted 22 January 2021; Published 11 February 2021

Academic Editor: James J. McGuirk

Copyright (C) 2021 Yonglei Qu et al. This is an open access article distributed under the Creative Commons Attribution License, which permits unrestricted use, distribution, and reproduction in any medium, provided the original work is properly cited.

\begin{abstract}
The performance of turbomachinery blade profiles, at low Reynolds numbers, is influenced by laminar separation bubbles (LSBs). Such a bubble is caused by a strong adverse pressure gradient (APG), and it makes the laminar boundary layer to separate from the curved profile surface, before it becomes turbulent. The paper consists on a joint experimental and numerical investigation on a flat plate with adverse pressure gradient. The experiment provides detailed results including distribution of wall pressure coefficient and boundary layer velocity and turbulence profiles for several values of typical influencing parameters on the behavior of the flow phenomena: Reynolds number, free stream turbulence intensity, and end-wall opening angle, which determines the adverse pressure gradient intensity. The numerical work consists on carrying out a systematic analysis, with Reynolds Average NavierStokes (RANS) simulations. The results of the numerical simulations are critically investigated and compared with the experimental ones in order to understand the effect of the main physical parameters on the LSB behavior. For RANS simulations, different turbulence and transition models are compared at first to identify the adaptability to the flow phenomena; then, the influence of the three aforementioned parameters on the LSB behavior is investigated under a typical aggressive adverse pressure gradient. Boundary layer integral parameters are discussed for the different cases in order to understand the flow phenomena in terms of flow time-mean properties.
\end{abstract}

\section{Introduction}

The performance of blade profiles for turbomachinery components is strongly influenced by many aerodynamic phenomena. Among them, laminar separation bubble is of primary importance for low-pressure turbine and compressor (LPT and LPC) applications, especially when high-lift profiles operating at low Reynolds number conditions are considered. Hence, the aerodynamic design of LPT and LPC airfoils needs an accurate prediction of the transition onset as well as of the separation extension in order to avoid the risk of performance deterioration due to the flow separation, which may occur at low Reynolds numbers.

LSB is caused by a strong adverse pressure gradient, and it makes the laminar boundary layer to separate from the curved profile surface; hence, it can have large, negative aerodynamic effects. Generally, additional drag will be induced due to the displacement of the outer flow, which results in decreased suction over the forward portion of profile and reduced pressure recovery in the rear parts. The key influence factor to the increasing of pressure drag is the geometry parameter of the LSB, especially its thickness in the normal direction to the profile surface. Moreover, a more dramatic effect will occur once the transition process in the separated shear layer is relatively slow and the adverse pressure gradient is relevant. Proceeding to the next step, turbulent momentum transport is not sufficient to close the bubble and a large separation occurs that extends right to the trailing edge. This causes a sudden loss of lift and a strong increase of drag along with significant hysteresis effects of force coefficients with varying angle of attack [1].

A large number of research works are carried out in order to systematically investigate the mechanism of the separation 
in laminar boundary layer flow, including theoretical researches, experiments, and numerical simulations $[2,3]$.

In recent years, the development of PIV testing technology, LES, and DNS simulation algorithms provided researchers with methods to understand the main dynamics driving the unsteady behavior of separated flows. Simoni et al. [4] and Marxen and Henningson [5] carried out relevant experimental and numerical simulation studies, and the results show that the shear layer is unstable due to a Kelvin-Helmholtz (K-H) process, leading to roll-up vortices. Particularly, after the separation of the flow, the separated shear layer induced by the inviscid $\mathrm{K}-\mathrm{H}$ instability process amplifies the fluctuations of the velocity. Once the separation bubble reaches the position of the maximum value of the displacement, the saturation of the fluctuations also occurs. Then, the large-scale vortical structures shed by the bubble initiate transition; the mixing process is strengthened by both coherent and small-scale structures, contributing to the boundary layer reattachment (if any). While the dynamic behavior of laminar separation bubbles has been intensively studied, the effects of flow parameters on separation flow process and its mechanism still need to be further understood.

By using different methods including the amplification of the velocity fluctuations, the response of laminar separation bubbles to external disturbances, the spanwise growth rate, and wall-normal amplitude of different modes, a large number of research works have been carrying out. For the problem of the amplification of the velocity fluctuations in the fore part of separated flow, investigations from Marxen et al. [6], Häggmark et al. [7], and Yarusevych et al. [8] show that it is typically well predicted by Linear Stability Theory (LST), which is in good agreement with the experimental results. Dovgal et al. [9] reported the stability characteristics of different mean velocity profiles. The theoretical and experimental research works made by Diwan and Ramesh [10] which focus on the wall distance effects on the most unstable frequency clearly show that when the distance from the wall is far enough, the wall effects vanish and both maximum amplified frequency and growth rate approach the values predicted for a free-mixing layer. For the problem of the response of laminar separation bubble to external disturbances, experiments and numerical simulations are carried out by means of controlling upstream perturbing waves. Among them, the investigations carried out at the Stuttgart University provide one of the most comprehensive views of the influences due to different parameters of the perturbing waves. Maucher et al. [11] presented the response of a laminar separation bubble to monochromatic waves, which mean simply composed wave trains, at the most unstable frequency with different amplitudes, and pointed out that the growth rate and the saturation level of the separation bubble are poorly affected. That means the main influence factor to the overall bubble size is the initial wave amplitude. Similar conclusions are reached in the DNS investigations of Jones et al. [12]. Using the Fourier-based decomposition method, Marxen et al. and Lang et al. [13] processed the experimental data from PIV and LDV measurements, in order to identify the growth rate in stream wise and wall-normal amplitude of different modes. The results show that the attached part of boundary layer is mainly affected by the spanwise wave, since the amplitude of corresponding modes is larger than those standing for the most unstable frequency of the shear layer. Lang et al. [14] also investigated the influence due to the superposition of spanwise wavenumbers, simulating the presence of streaky structures, to the frequency of unstable shear layer. The investigations mentioned above show the overall view about the development of monochromatic waves. In reality, the unstable source is superposed with a multitude of waves with different amplitude, which should further contribute to the understanding of the dynamics driving laminar separation bubbles.

Relevant investigations are carried out by the enhancement of high-resolution numerical simulations recently, which focus on separated flow and transition characteristics under varied conditions. LES study of a transitional separated boundary layer over a flat plate with a semicircular leading edge at two freestream turbulence levels $(<0.2 \%$ and $5.6 \%$ at leading edge) is presented by Langari and Yang [15]. The flow visualizations and spectral analysis of the separated shear layer reveal that the 2D Kelvin-Helmholtz instability mode is bypassed at higher levels leading to earlier breakdown to turbulence. Li and Yang [16] investigated numerically the transition process in a separation bubble induced by an adverse pressure gradient on a flat plate with an elliptical leading edge under $2.9 \%$ freestream turbulence intensity by LES. The results suggest that the transition process is more rapid and over a shorter streamwise distance due to the dominant role played by the streaks, and the usual secondary instability stage under low freestream turbulence intensity where very coherent $2 \mathrm{D}$ spanwise rolls get distorted gradually and eventually broken into 3D structures has been bypassed. In a review research [17], it demonstrates that at elevated freestream turbulent intensity, separation could be eliminated completely and flow remains attached, that is, bypass transition could occur in geometrically induced separation bubbles. The present work will make a comparative analysis of this phenomenon. LES study of a transitional separated bubble over a flat plate with a blunt leading edge at a very low freestream turbulence level is carried out by Yang et al. [18], with result indicates that a three-dimensional secondary instability is the main mechanism at work whereas the subharmonic mode in the form of vortex pairing is hardly active. By using a high order, structured, multiblock, compressible Navier-Stokes solver, Sengupta et al. [19, 20] investigated the individual and cumulative effects of forced frequency oscillations, unsteady wakes, and freestream turbulence on the separation-induced transition caused by an adverse pressure gradient on a flat plate geometry.

Unlike the in-depth study of the dynamic behavior of the flow separation process, other previous works focused on determining the overall response of the laminar separation bubble without analyzing the dynamic control of the flow stability mechanism and related shedding phenomena. For example, Volino [21] studied the time-averaged structure characteristics of laminar separation bubbles by hot-wire measurements and found that with the increasing of Reynolds number, the characteristic scale of laminar separation 
bubble became smaller. Similar results can be found when the freestream turbulence intensity is increased. The study of Yaras [22] shows that for the plate flow with strong adverse pressure gradient, the relationship between the characteristic points of separation can be constructed by formulas, which means that it is possible to predict the overall structure of separation bubble by using Reynolds number, turbulence intensity, and integral scale as variables. Other formulas have been constructed and widely used in the field of turbomachinery [23].

The present paper mainly focuses on understanding the effects of the overall flow parameters on LSB from the RANS analysis of the time-averaged experimental results. The flat plate boundary layer experiment corresponds to the working environment of a high-lift blade profile in turbomachinery under variable Reynolds number conditions. The Reynolds number ranges from 150000 to 500000 , and the turbulence intensity varies from $0.65 \%$ to $5.0 \%$. The adverse pressure gradient includes two conditions: the upper and lower endwall angles of 7-2.5 degrees and 12-7 degrees (briefly named by D7-2.5 and D12-7 below), respectively. The fixed turbulence length scale adopted is $8 \mathrm{~mm}$. The experiments provide several parameters, including the detailed results of wall pressure coefficient, boundary layer velocity, and turbulence profiles. These quantities are also studied and validated by numerical simulations from the perspectives of timeaveraged state.

\section{Test Section and Measuring Techniques}

The experiments are carried out in an open-loop low-speed wind tunnel of the Aerodynamics and Turbomachinery Laboratory of the University of Genova. The main structure of the test section which includes a flat plate with an elliptical leading edge and two end-walls with adjustable horizontal angle is shown as Figure 1. The distance from the leading edge to the reference point of Reynolds number is $300 \mathrm{~mm}$, and the 2-dimensional measuring plane is located at midspan of the plate. The upper and lower end-walls can rotate to adjust the angle of attack that stands for the required adverse pressure gradient. To survey the proper attack angle of the end-walls, several pressure taps are set both on the top and the bottom sides of the leading edge. Other pressure taps are distributed along the surface of the plate in order to obtain the surface pressure distribution. Upstream of the test section, the required turbulence intensity is generated by placing grid plates of different scales.

Measurements are performed with complementary techniques, including hot-wire (HW) anemometry, Laser Doppler Velocimetry (LDV), and Particle Image Velocimetry (PIV). The analysis of the data obtained from the complementary measuring techniques helps us to understand the instability mechanisms involved in the transition/reattachment processes of the separated shear layer in details. The use of high-precision LDV allows investigating reverse flow magnitude and both Reynolds normal and shear stress distributions along the separated flow region, while the amplification process of flow oscillations induced by instability mechanisms can be investigated by HW anemometer with

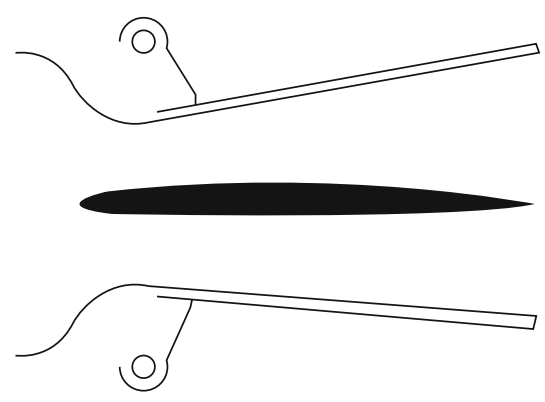

FIgURE 1: Test section structure.

TABle 1: Comparison of grid dependency.

\begin{tabular}{lccc}
\hline & Mesh 1 & Mesh 2 & Mesh 3 \\
\hline Number of elements & 240000 & 360000 & 540000 \\
Mass flow rate $\left(10^{-3} \mathrm{~kg} / \mathrm{s}\right)$ & 1.678 & 1.742 & 1.743 \\
\hline
\end{tabular}

high-frequency response performance. The instantaneous velocity vector maps given by PIV results complement the flow field analysis, providing information on the generation and evolution of the large-scale coherent structures shed as a consequence of the separated shear layer roll-up.

\section{Computational Domain and Numerical Model}

The experimental test section is modelled in order to carry out several numerical simulations. For the mesh generation, Numeca IGG [24] is employed in order to construct a structured mesh. The computational domain consists of proper extensions both upstream and downstream of the flat plate region. Considering that the computational domain structure has typical characteristics of external flow field case, a C-type topology is adopted. The height of the first cell near the solid walls is chosen in order to ensure a value of the nondimensional wall coordinate, $y^{+}$, close to one (obviously for the higher tested Reynolds number), and the viscous layer is generated with appropriate growth rate to ensure to capture the boundary layer characteristics. Based on the above principles, three computational grids with different number of elements are generated in order to evaluate the solution's grid dependency. The value of mass flow rate is employed as the test variable parameter and the results are reported in Table 1. The comparison shows that no appreciable differences are reported for mesh 2 and mesh 3; hence, the intermediate refined mesh is adopted. Figure 2 shows the sketch of the employed mesh, with focus on the leading edge zone. Figure 3 also illustrates the grid dependency by comparing the velocity profiles along the plate surface in three normalized feature positions under typical case (D12-7 Re150000 Tu1. 5\%, which will be explained detailed next). It also shows no substantial distinction among the three meshes.

The numerical simulations are carried out with the software Ansys Fluent [25]. The solution method is SIMPLEC scheme, while the least square cell based is adopted for the 


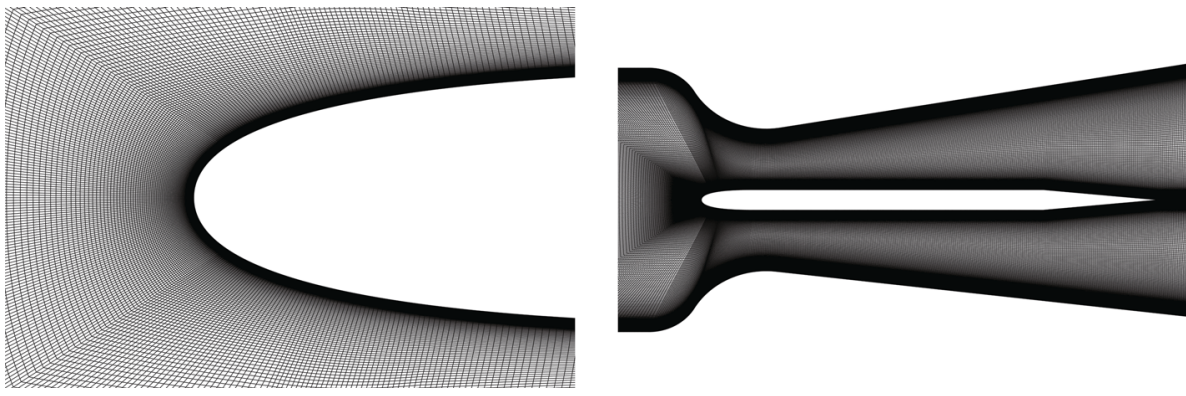

FIgURE 2: Mesh generation sketch.
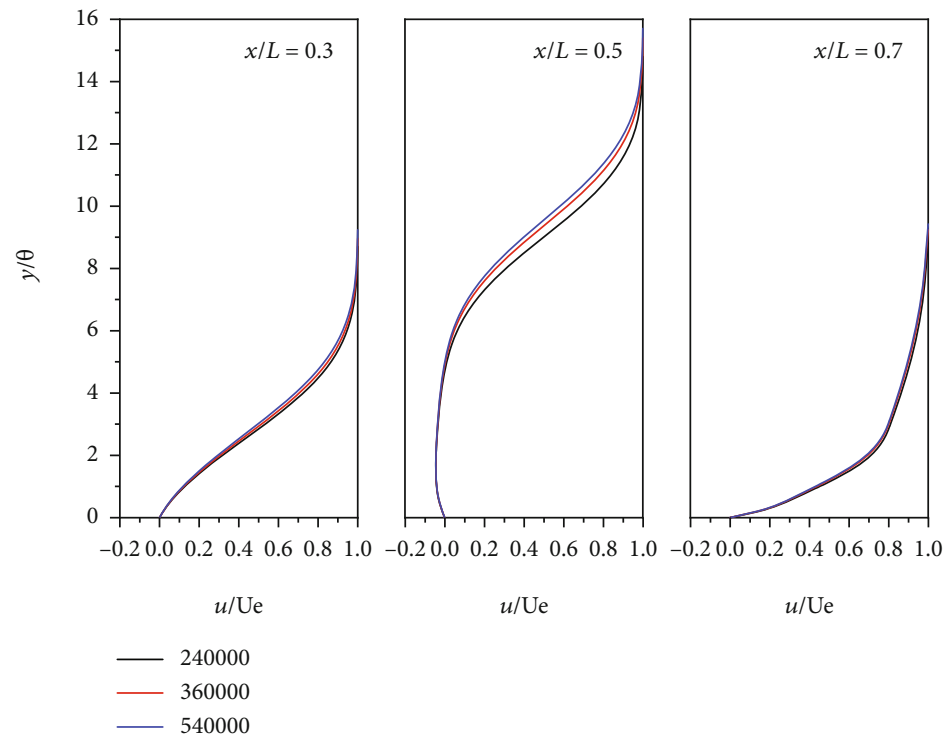

FIGURE 3: Grid dependency checking by velocity profiles in typical condition.

TABle 2: Boundary conditions.

\begin{tabular}{lcccc}
\hline Name & Inlet & Outlet & Flat plate & End-wall \\
\hline Type & Total pressure & Static pressure & Ordinary wall & Eulerian wall \\
\hline
\end{tabular}

spatial discretization. The boundary conditions set for the tested cases are reported in Table 2. In both inlet and outlet sections, total pressure is imposed. For turbulence and transition modelling, several models are tested in order to identify the most proper one to describe the flow behavior within the domain and in particular along the flat plate solid wall, as reported in the following paragraph.

\section{Transition Models}

The standard $\mathrm{k}-\varepsilon$ model is a semiempirical model. In the derivation of the model, it is assumed that the flow is completely turbulent and the effect of molecular viscosity can be neglected. Therefore, the standard $\mathrm{k}-\varepsilon$ model can only be applied to a complete turbulence, i.e., high Reynolds number conditions, or freestream far away from the wall. For this reason, turbulence models have been developed, which are suitable for the near-wall region and have the resolution of laminar-turbulent transitional progress. To predict the flow transition, a variety of mathematical models have been proposed and developed in the past for numerical simulations.

By optimizing the k- $\omega$ 2-equation model, Menter et al. proposed a turbulence model, named by Baseline (BSL) model, similar to Wilcox model $[26,27]$ but avoiding its strong freestream sensitivity. Also, k- $\omega$ Shear Stress Transport (k- $\omega$ SST) model, which a modified definition of the eddy-viscosity with respect to BSL model, and taking into account of the effect of the transport of the principal turbulent shear stress was also proposed [28]. Later, Menter et al. developed the Transition SST (T SST) model [29, 30] for unstructured grids and massive parallel execution, validated by applying it to turbomachinery and aerodynamic test cases. 
By introducing a third transport equation to predict the lowfrequency velocity fluctuation in the boundary layer before the transition, which has been determined as the precursor of the transition, Walters and Cokljat [31] developed Transition $\mathrm{k}-\mathrm{kl}-\omega(\mathrm{T} \mathrm{k}-\mathrm{kl}-\omega)$ model, testing and validating it on airfoils and flat plate boundary layers with and without applied pressure gradient cases. The test cases' results demonstrated the ability of the model of successfully representing transitional flow behavior with a reasonable degree of accuracy.

The T SST model was proposed by Menter et al. [29] and has been improved continuously. Its main feature is to summarize and improve the transition criterion and equations. Among them, the first equation is still intermittency transport equation, which can be used to induce the transition process. The equation is optimized and generalized on the basis of the original one. The second transport equation avoids the increase of nonlocal information introduced by the quantities used in the experimental correlations. Correlations are mainly based on freestream data, such as turbulence intensity or pressure gradient outside the boundary layer. The additional equation is established by using the Reynolds number of transition occurrence $\operatorname{Re}_{\theta t}$. The validated model was integrated by Ansys Fluent and named Transition Shear Stress Transport model, also known as $\left(\gamma-\operatorname{Re}_{\theta}\right)$ model. Like known to us, the model is based on the coupling of the k- $\omega$ SST transport equations with two other transport equations, one for the intermittency and one for the transition onset criteria, in terms of momentum-thickness Reynolds number. Hence, it is actually a four-equation model.

In the T SST model, the transport equations of turbulence kinetic energy $k$ and specify dissipation rate $\omega$ are still resolved, and all terms are the same as k- $\omega$ SST model, except for three terms, including the production $\left(P_{k}\right)$, the diffusion $\left(D_{k}\right)$, and the blending function $F$. In the production term, $P_{k}$ is multiplied by the turbulence intermittency $\gamma$, which is the percentage of time that turbulent fluctuations are present in the boundary layer. By means of taking values from 0 to 1 , the intermittency states the flow from laminar to fully turbulent. Hence, intermittency is damping the production of turbulence where the boundary layer is laminar and transitional. For the dissipation term $D_{k}$, it is replaced with

$$
D_{k} \times \min (\max (\gamma, 0.1), 1.0)
$$

Hence, it is another limiter that ensures that the dissipation does not drop below $10 \%$ of turbulent value. At the same time, the wall still damps turbulence even when the flow is laminar. While for the blending function $F_{1}$, it is corrected to prevent to be zero in the laminar boundary layer, which is expressed as followed, to make sure the model will not switch to the k-E model in the laminar boundary layer.

In a laminar boundary layer, the $k$ equation is

$$
\frac{\partial(\rho k)}{\partial t}+\frac{\partial\left(\rho U_{j} k\right)}{\partial x_{j}}=\frac{\partial}{\partial x_{j}}\left[\left(\mu+\frac{\mu_{t}}{\sigma_{\gamma}}\right) \frac{\partial k}{\partial x_{j}}\right]-0.1 D_{k}
$$

In a turbulent boundary layer, the $k$ equation is

$$
\frac{\partial(\rho k)}{\partial t}+\frac{\partial\left(\rho U_{j} k\right)}{\partial x_{j}}=\frac{\partial}{\partial x_{j}}\left[\left(\mu+\frac{\mu_{t}}{\sigma_{\gamma}}\right) \frac{\partial k}{\partial x_{j}}\right]+P_{k}-D_{k}
$$

The transport equation for the intermittency $\gamma$ is defined as follows:

$$
\frac{\partial(\rho \gamma)}{\partial t}+\frac{\partial\left(\rho U_{j} \gamma\right)}{\partial x_{j}}=P_{\gamma 1}-E_{\gamma 1}+P_{\gamma 2}-E_{\gamma 2}+\frac{\partial}{\partial x_{j}}\left[\left(\mu+\frac{\mu_{t}}{\sigma_{\gamma}}\right) \frac{\partial \gamma}{\partial x_{j}}\right],
$$

while the transport equation for the transition momentum thickness Reynolds number is

$$
\frac{\partial\left(\rho \widetilde{\operatorname{Re}}_{\theta t}\right)}{\partial t}+\frac{\partial\left(\rho U_{j} \widetilde{\operatorname{Re}}_{\theta t}\right)}{\partial x_{j}}=P_{\theta t}+\frac{\partial}{\partial x_{j}}\left[\sigma_{\theta t}\left(\mu+\mu_{t}\right) \frac{\partial \widetilde{\operatorname{Re}}_{\theta t}}{\partial x_{j}}\right] .
$$

$\mathrm{T} \mathrm{k}-\mathrm{kl}-\omega$ model is a new version of single-point eddyviscous turbulence model with three additional transport equations. It closes the relevant terms in the model based on physical phenomena rather than empirical methods and discusses that these terms should have a reasonable form. The additional third transport equation includes the prediction of the low-frequency velocity fluctuation vector modulus in the pretransition boundary layer. The model has been validated by a large number of numerical examples and applied to commercial computational fluid dynamics software. It has been applied to a large number of related cases, including plate boundary layer flow with or without pressure gradient, and airfoil cases with different geometric characteristics, Reynolds number, turbulence level of incoming flow, and angle of attack. These test cases prove that the model has the ability to successfully reproduce the transition flow behavior and has reasonable accuracy, especially for the compressible flow problems that cannot be predicted by the conventional model for laminar-turbulent transition development [31].

The concept of laminar energy $k_{L}$ is adopted in the model, which is summarized by instability mechanism and transition mechanism in the process of studying laminar boundary layer. The theoretical basis of T k-kl- $\omega$ model is that the transition process itself is accomplished by energy transfer from laminar kinetic energy $k_{L}$ to turbulent kinetic energy $k_{T}$. Variables $k_{T}$ are used to characterize the modes of fluctuations to show the characteristics of complete turbulence, such as strong three-dimensional, multiscale and timescale, energy spectrum, and significant viscous dissipation. Initialization of the transition process is based on local (single point) flow conditions, and a shear shielding concept is adopted to suppress the nonlinear turbulence breakdown mechanism. At the same time, the method of transition initiation considering the time scales of nonlinear disturbance amplification and dissipation correlation is used to predict more accurately the effect of free turbulence length scale on the transition process. 
The T k-kl- $\omega$ model is considered to be a three-equation eddy-viscosity type, which includes transport equations for turbulent kinetic energy $\left(k_{T}\right)$, laminar kinetic energy $\left(k_{L}\right)$, and the inverse turbulent time scale $(\omega)$. The transport equations of T k-kl- $\omega$ model are given as the following. It is based on incompressible condition, with ignoring the volume force.

$$
\begin{gathered}
\frac{D k_{T}}{D t}=P_{k_{T}}+R_{B P}+R_{N A T}-\omega k_{T}-D_{T}+\frac{\partial}{\partial x_{j}}\left[\left(v+\frac{\alpha_{T}}{\alpha_{k}}\right) \frac{\partial k_{T}}{\partial x_{j}}\right], \\
\frac{D k_{L}}{D t}=P_{k_{L}}-R_{B P}-R_{N A T}-D_{L}+\frac{\partial}{\partial x_{j}}\left[\nu \frac{\partial k_{L}}{\partial x_{j}}\right] \\
\frac{D \omega}{D t}=C_{\omega 1} \frac{\omega}{k_{T}} P_{k_{T}}+\left(\frac{C_{\omega R}}{f_{w}}\right) \frac{\omega}{k_{T}}\left(R_{B P}+R_{N A T}\right)-C_{\omega 2} \omega^{2} \\
+C_{\omega 3} f_{\omega} \alpha_{T} f_{w}^{2} \frac{\sqrt{k_{T}}}{d^{3}}+\frac{\partial}{\partial x_{j}}\left[\left(v+\frac{\alpha_{T}}{\alpha_{k}}\right) \frac{\partial \omega}{\partial x_{j}}\right] .
\end{gathered}
$$

Due to the limitation of article length, the specific definitions and correlation quantities in the equations above, as well as the specific values of the coefficients, were used (please refer to the reference [25]).

In present work, three turbulence models are employed and results are compared with experimental ones, including a traditional turbulence model (k- $\omega$ SST) and two turbulence models with transition criterion ( $\mathrm{T} \mathrm{k}-\mathrm{kl}-\omega$ and $\mathrm{T}$ SST). Figures 4 and 5 show the distribution of pressure coefficients obtained by the three models under two conditions, characterized by different flow conditions (Reynolds number and turbulence intensity) and different opening angles of the diffusing test section. The comparison shows that the three models have different reactivity to flat plate LSB phenomena. In these two figures, both the pressure distribution on the plate surface and the LSB region behavior reflected by the T $\mathrm{k}-\mathrm{kl}-\omega$ model have the highest consistency with the experimental results. Especially in Figure 4, the T k-kl- $\omega$ curve begins to separate at $x / L=0.52$, then reaches the maximum value of separation thickness at $x / L=0.79$, finally reattaches at the position $x / L=0.91$. These points are close to the experimental ones, which means the bubble detachment and length are well predicted. In Figure 5, the end-walls' angle and Reynolds number increase. The detachment point and the growth trend of the separation zone obtained by the $\mathrm{T}$ $\mathrm{k}-\mathrm{kl}-\omega$ model are still the closest to the experimental results. However, the positions of the maximum thickness of LSB and the reattachment point are located forward with respect to experimental ones. Thus, the bubble length is shorter than in the experiment.

The pressure distribution curves obtained by $\mathrm{T}$ SST model are similar to those of $\mathrm{T} \mathrm{k-kl- \omega}$ model, but the deviation from the experiment is slightly bigger than that of the latter. Furthermore, in Figure 5, the location of maximum separation thickness and the reattachment point are ahead of the latter, hence the shorter LSB's length. For k- $\omega$ SST model, as expected, the turbulence model (without transition model) is not able to correctly predict the flow behavior. In Figure 4, the deviation and differences from the experiment

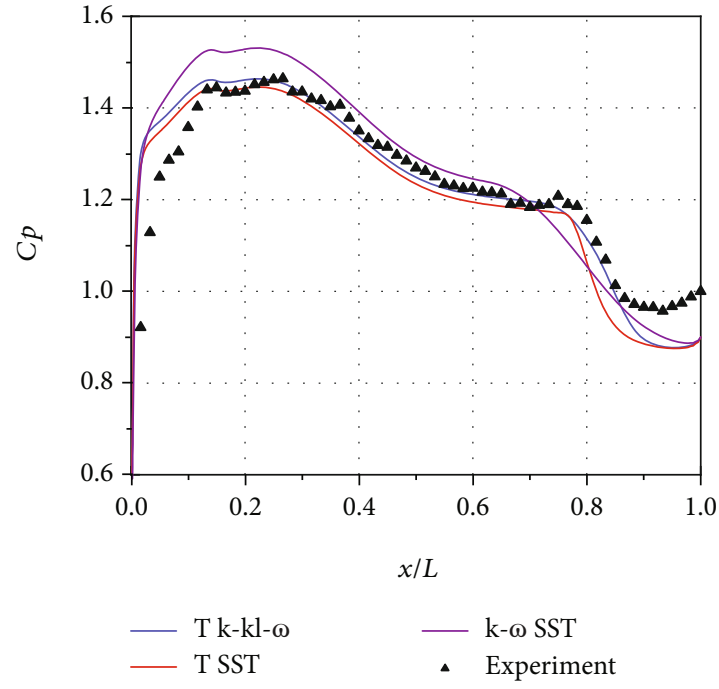

Figure 4: Turbulence model comparison in case D7-2.5 $\mathrm{Re}=$ $150000 \mathrm{Tu}=0.65 \%$.

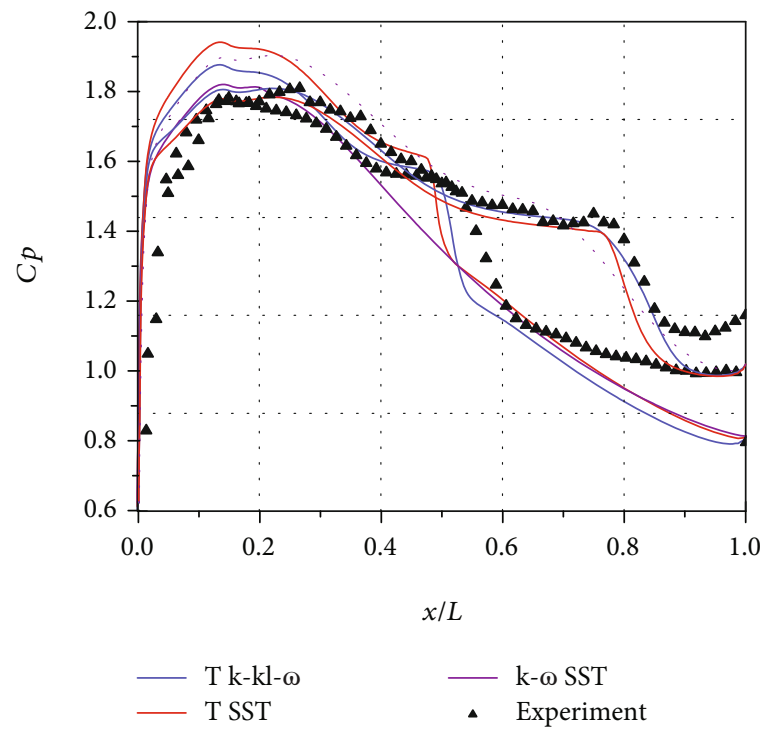

FIGURE 5: Turbulence model comparison in case D12-7 Re $=300000$ $\mathrm{Tu}=1.5 \%$.

are the most obvious. From the appearance point of view, the thickness of the separation bubble changes most smoothly, while the position of the LSB's maximum thickness moves forward by $0.1 x / L$, forming a flat and longer LSB. In Figure 5, the k- $\omega$ SST model cannot reflect the existence of LSB, with a flat pressure coefficient distribution curve. According to the above analysis, the T k-kl- $\omega$ model can fully reflect the behavior of LSB, which has the most similar appearance to the experiment. Therefore, the $\mathrm{T} \mathrm{k}-\mathrm{kl}-\omega$ model is used in subsequent calculation and analysis.

\section{Results and Discussion}

To validate the compatibility of the numerical scheme, a systematical comparison and analysis with the specific case 


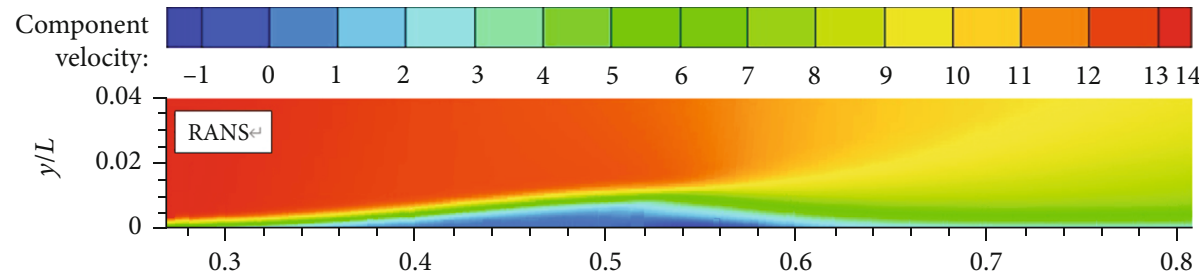

(a)

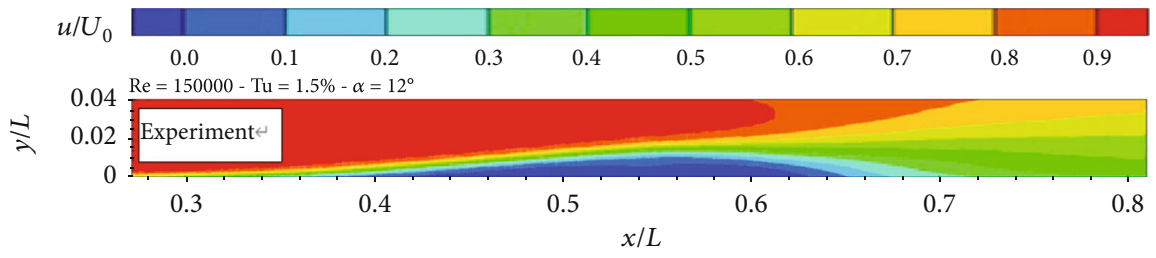

(b)

Figure 6: Color contour of velocity component $u$ ((a) simulation and (b) experiment).

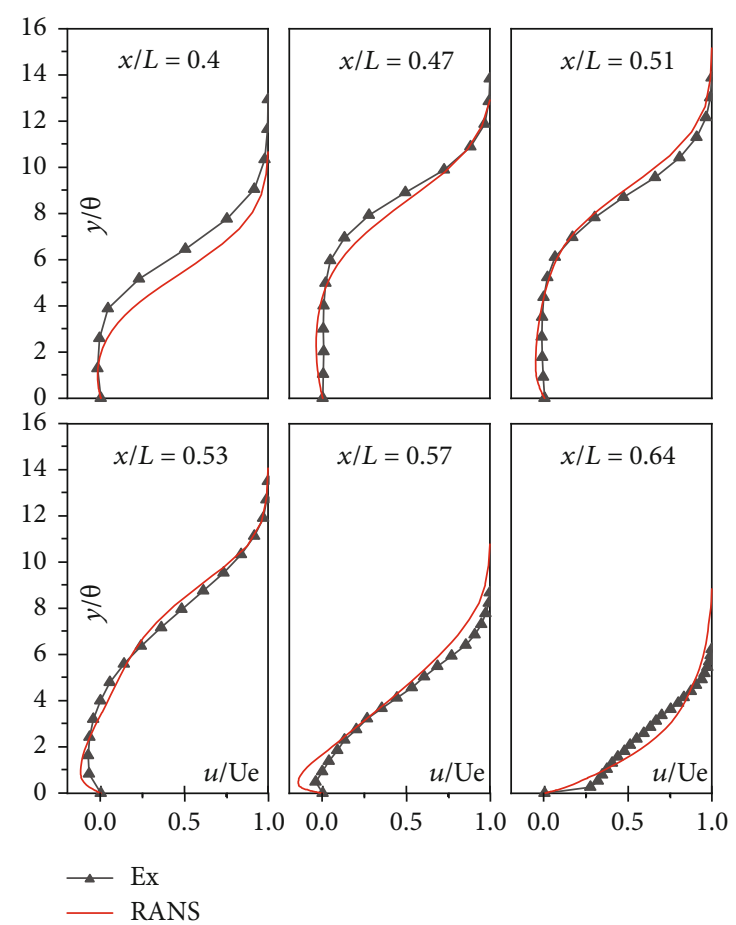

FIGURE 7: Velocity profile comparison along the plate surface.

between experiment and simulation was carried out. Figures 6 and 7 show the results for the case D12-7, with Reynolds number and turbulence intensity equal to 150000 and $1.5 \%$, respectively. As mentioned before, the $\mathrm{T} \mathrm{k}-\mathrm{kl}-\omega$ transition model is employed.

Figure 6 shows that the general LSB's behavior of both experiment and simulation is very similar. There is no substantial difference between the two sets of LSB's feature points. Experimentally, the separation point locates at about $x / L=0.36$, while the simulation predicts this position at $x / L$ $=0.35$. The bubble maximum thickness is captured at $x / L$ $=0.56$ and $x / L=0.52$, for the experiment and simulation, respectively. After the position of the maximum thickness, the LSBs begin to reattach, at $x / L=0.65$ for the experiment, while the numerical simulation provides a value of $x / L=$ 0.62. Although the characteristic points do not coincide completely, the development trends of separation bubbles are the same even if the overall length and thickness of LSB corresponding to the experiment are slightly larger than the numerical simulation ones.

In Figure 7, the velocity profile comparisons along the plate surface in specific positions between experiment and numerical simulation are plotted. The curves are normalized by the local velocity of the main flow. For the positions ahead of the maximum thickness $(x / L=0.41$ and $x / L=0.47)$, the experimental curves show a thicker separated flow area than those of numerical simulation. In the three subfigures of the first row, the region with velocity component $u$ less than 0 increases gradually in the direction perpendicular to the plate surface, and the magnitude values increase gradually, which reflects the growth of the reverse flow region and the enhancement of the internal reflux in the separation bubble. In the subfigures corresponding to $x / L=0.53$ and $x / L=0.57$, the LSB starts the reattachment process, and the comparison with the simulations becomes greater. The velocity profiles near the reattachment point are shown in position $x / L=$ 0.64 , and the numerical simulation result reflects the timeaveraged characteristic of turbulent boundary layer.

A systematic numerical simulation is made with three factors including Reynolds number, turbulence intensity, and end-wall opening angle variation, in order to obtain the response of the LSB to these parameters. Figure 8 gives a comprehensive comparison of dimensionless velocity component distribution. Here, the local velocity component $u$ is normalized by the mainstream velocity of the inlet section for each case, while the horizontal and vertical coordinates are normalized by the length of the plate. For all the 12 conditions, the Reynolds number and the turbulence intensity increase from top to bottom and from left to right, respectively. Therefore, the most extreme cases occur in the upper left and lower right positions accordingly. In each subfigure, the obvious difference of velocity component $u$ distribution can be observed: there is a closed dark blue part in the center 


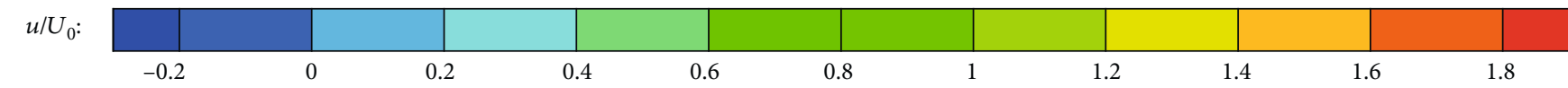
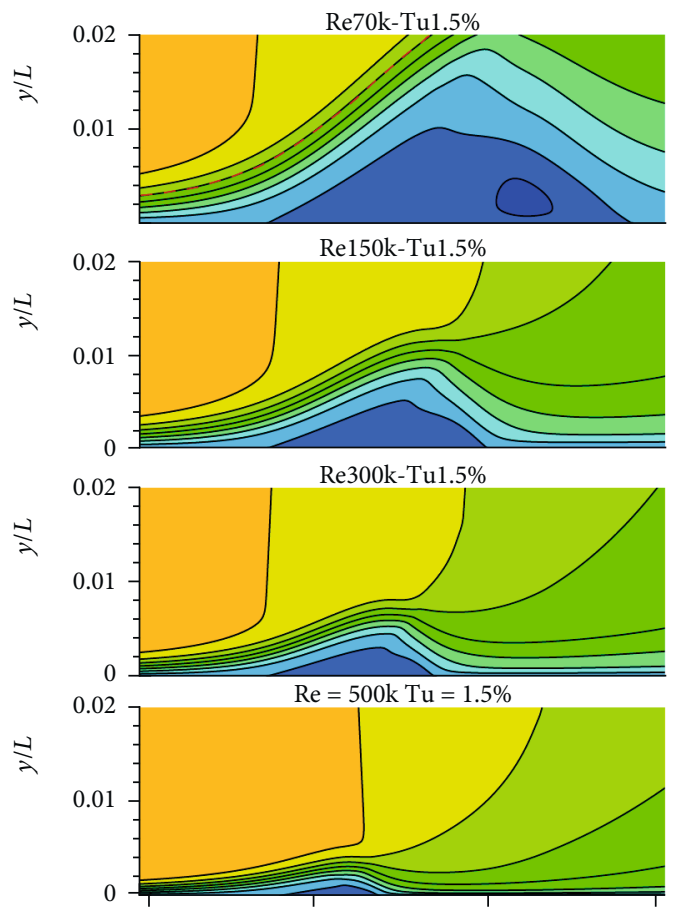

0.2

0.4

0.6

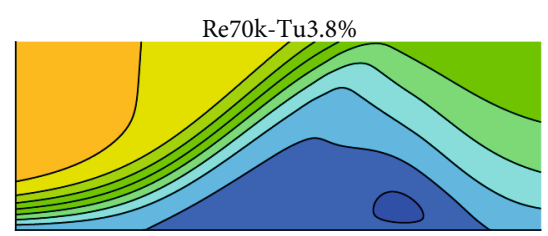

Re150k-Tu3.8\%

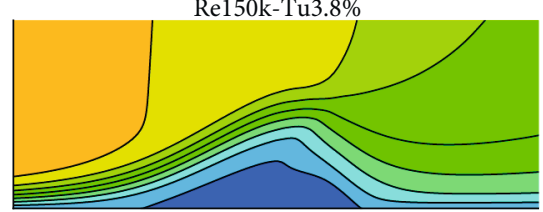

Re300k-Tu3.8\%
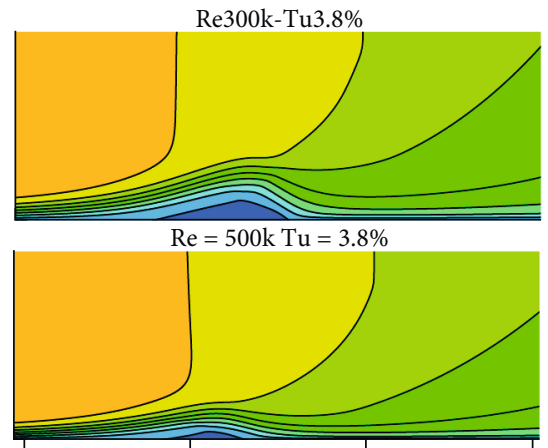

0.4

$0.8 \quad 0.2$

$x / L$

$x / L$

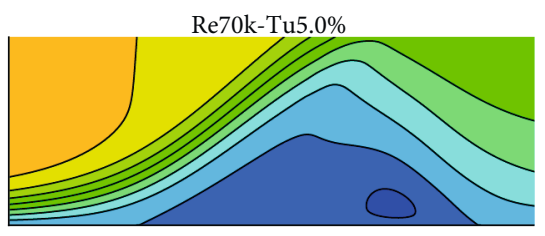

Re150k-Tu5.0\%

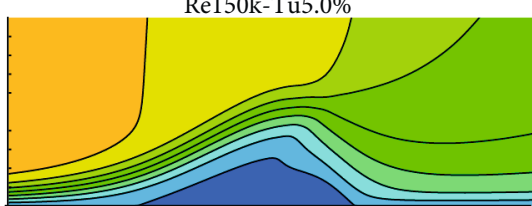

Re300k-Tu5.0\%
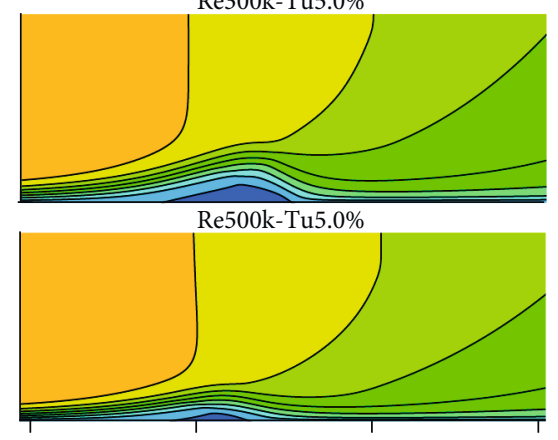

$\begin{array}{ll}0.8 & 0.2\end{array}$

0.4

$x / L$

FIGURE 8: Comparison of normalized velocity component $u / U_{0}$.

of the region near the wall, i.e., the reversed flow opposite to the mainstream velocity. Outside the region, both the direction and magnitude of velocity gradually return to the mainstream state. This closed reverse flow region, which is confined to a certain area near the wall, forms the laminar separation bubble defined earlier.

In the subfigure of the upper left corner, the boundary of $u / U_{0}=1$ is marked with a red dotted line, which can help to understand the variation of velocity component $u$. It is found that with the increases of Reynolds number and turbulence intensity, the range of closed recirculation zone, the LSB, is significantly reduced. The preliminary results show that the increase of these two flow parameters can inhibit the strength of laminar separation bubbles, but obviously, with a distinguished influence level: the trend of LSB decrease in all subfigures of each column is larger than that in each row, that is, the influence of Reynolds number change in freestream is stronger than that of turbulence intensity. The comprehensive comparison also reveals another phenomenon: although the range and strength of laminar separation bubbles are affected by the increase of flow parameters, the initial position of laminar separation bubbles does not fluctuate in a wide range in each case, while the positions where separation ends and the maximum thickness onsets vary significantly, which affects the structural characteristics of LSB.

A series of pressure coefficient $\left(C_{p}\right)$ distributions due to the variations of turbulence intensity and Reynolds number are plotted in Figures 9 and 10, with fixed opening angle of D12-7. The experimental results under the conditions of $\mathrm{Tu}$ $=1.5 \%$ and Reynolds number of 150000 and 500000, respectively, are taken as references. In the figure, also the peculiar positions highlighting the time-mean structure of the bubble are shown: "S" stands for the beginning of separation, "M" indicates LSB's maximum thickness, and " $R$ ” highlights the reattachment position.

$C_{p}$ distributions are compared in Figure 9 with two turbulence intensity levels, corresponding to the minimum and maximum values adopted in the experiments. It can be found that the feature point " $S$ " in each subfigure has the same abscissa, $x / L=0.35$, which means that the separation position barely shifts varying both Tu and Re. For the points " $M$ " and "R," as the Reynolds number increases, they move forward to the upstream of plate, i.e., in the subfigure of $\mathrm{Tu}$ $=1.5 \%$; the maximum thickness abscissa moves from $x / L$ $=0.62$ to $x / L=0.44$, while the reattachment one moves from $x / L=0.75$ to $x / L=0.5$. These changes diminish the LSB's dimension in length, with an obvious shrinking trend. A shortening distance between points " $M$ " and " $R$ " is also due to the Reynolds number increment, hence, the fore part and rear part of LSB response simultaneously to the variation of Re. In the condition of $\operatorname{Re}=500000$, the simulation curve is highly consistent to the experiment one.

The same $C_{p}$ data is reorganized according to the fixed Reynolds number in Figure 10, with minimum and maximum 


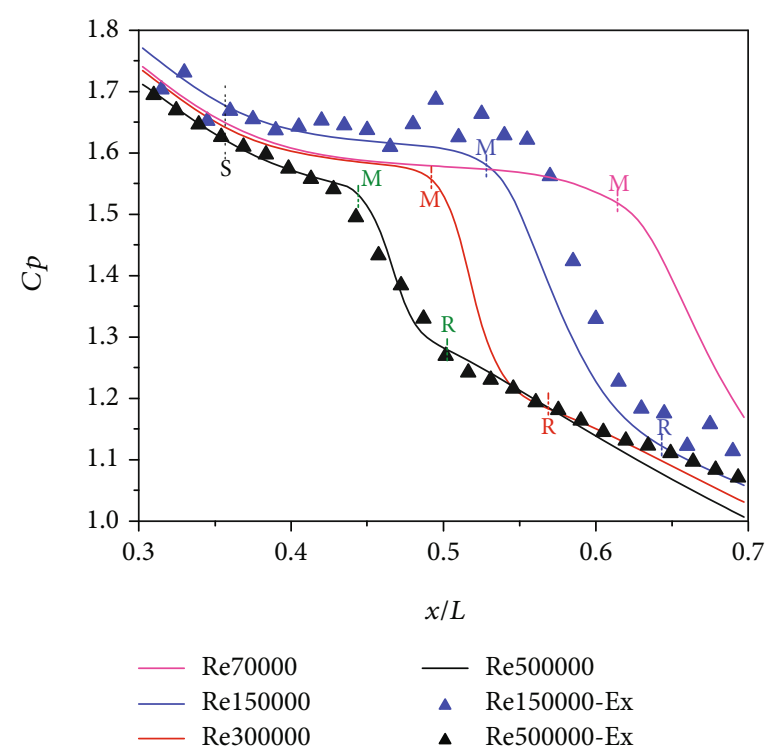

(a)

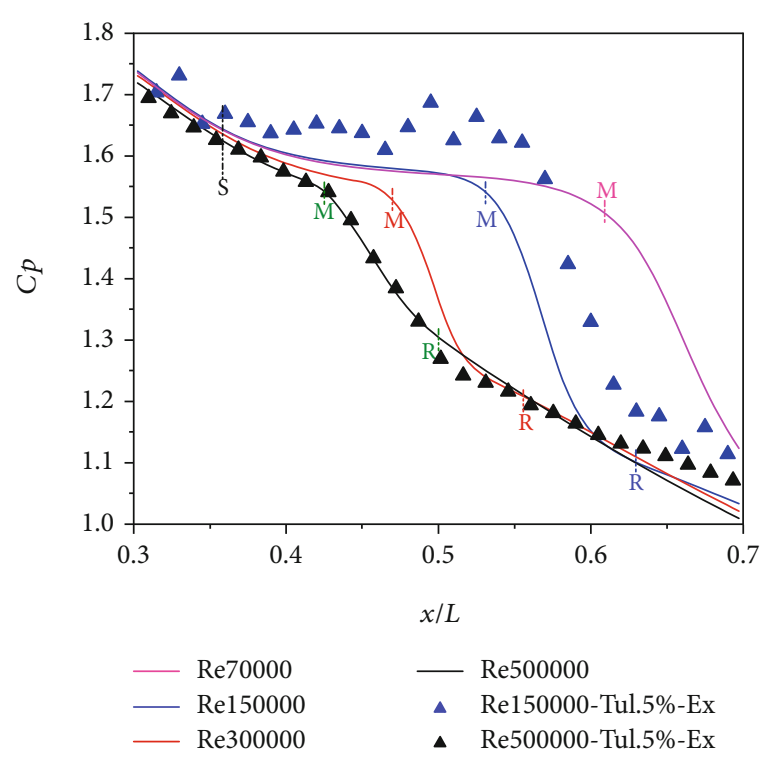

(b)

Figure 9: $C_{p}$ comparison with fixed (a) Tu1.5\% and (b) Tu5.0\%.

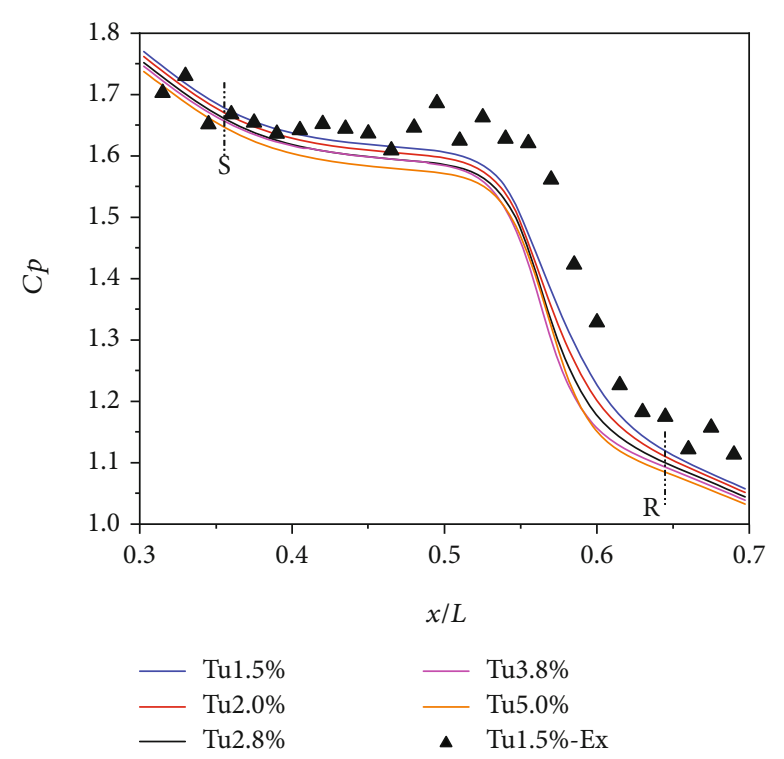

(a)

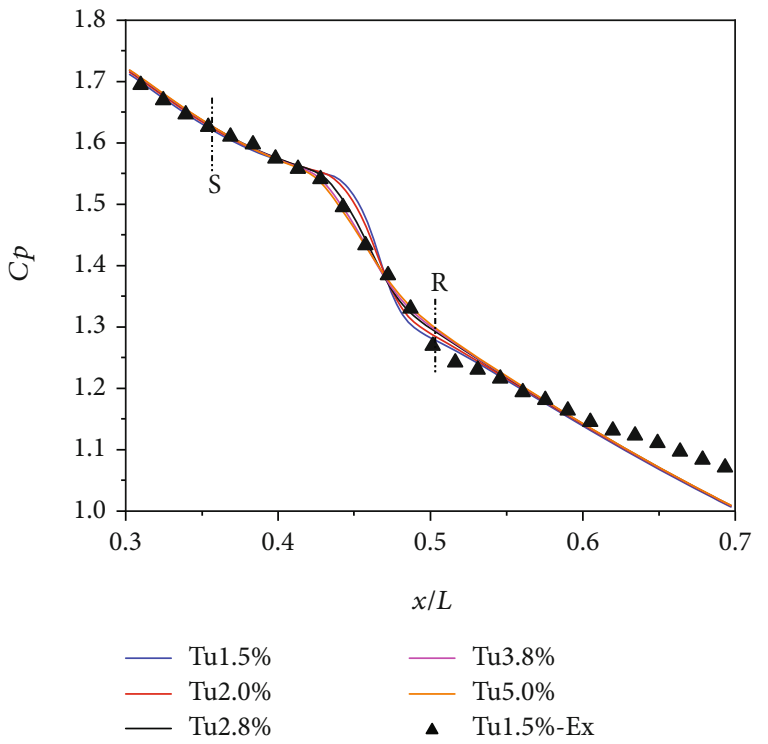

(b)

Figure 10: $C_{p}$ comparison with fixed (a) Re150000 and (b) Re500000.

Re values adopted in the experiments. By this way of classification, it can be seen that the variation of turbulence intensity has a weaker influence to the general behavior of LSB. The similar distributions of $C_{p}$ curve are plotted in each fixed $\mathrm{Re}$ condition. Furthermore, all the main points, including the separation position, maximum thickness position, and reattachment position, have a marginal change, hence indicating the same length scale of LSB. Compared with the two subfigures, when Reynolds number increases, the influence of turbulence intensity on separation bubbles decreases. However, the fore part of five $C_{p}$ curves in the right subfigure coincides fairly well. In the condition of long LSB, such as $\mathrm{Tu}=1.5 \%$ and $\mathrm{Re}$
$=150000$, the simulation curve has the same developing trend of the experimental one, although the difference between them enlarges. The detachment and reattachment positions are listed in Tables 3 and 4, respectively, with opening angle D12-7 and varied $\mathrm{Re}$ and $\mathrm{Tu}$, while position " $\mathrm{M}$ " is plotted in Table 5 as maximum $\delta^{*}$.

Figure 11 compares the velocity characteristics under fixed turbulence intensity conditions. It is observed that in the position of $x / L=0.3$, because the separation of flow has not yet occurred (referring to the distribution of the $C_{p}$ curve), the velocity profiles all show the same laminar flow characteristics, and the curves corresponding to different 
Table 3: Detachment positions $(x / L)$ with D12-7.

\begin{tabular}{lccc}
\hline & $\mathrm{Tu}=1.5 \%$ & $\mathrm{Tu}=3.8 \%$ & $\mathrm{Tu}=5.0 \%$ \\
\hline $\mathrm{Re}=70000$ & 0.35 & 0.35 & 0.35 \\
$\mathrm{Re}=150000$ & $0.35(0.36-\mathrm{Ex})$ & 0.35 & 0.35 \\
$\mathrm{Re}=300000$ & 0.35 & 0.36 & 0.37 \\
$\mathrm{Re}=500000$ & 0.36 & 0.38 & 0.38 \\
\hline
\end{tabular}

TABLE 4: Reattachment positions $(x / L)$ with D12-7.

\begin{tabular}{lccc}
\hline & $\mathrm{Tu}=1.5 \%$ & $\mathrm{Tu}=3.8 \%$ & $\mathrm{Tu}=5.0 \%$ \\
\hline $\mathrm{Re}=70000$ & 0.75 & 0.74 & 0.73 \\
$\mathrm{Re}=150000$ & $0.64(0.65-\mathrm{Ex})$ & 0.62 & 0.62 \\
$\mathrm{Re}=300000$ & 0.57 & 0.55 & 0.55 \\
$\mathrm{Re}=500000$ & 0.50 & 0.49 & 0.49 \\
\hline
\end{tabular}

TABle 5: Maximum $H_{12}$ positions $(x / L)$ with D12-7.

\begin{tabular}{lccc}
\hline & $\mathrm{Tu}=1.5 \%$ & $\mathrm{Tu}=3.8 \%$ & $\mathrm{Tu}=5.0 \%$ \\
\hline $\operatorname{Re}=70000$ & $0.58(0.55-\mathrm{Ex})$ & - & 0.56 \\
$\operatorname{Re}=150000$ & $0.51(0.51-\mathrm{Ex})$ & 0.51 & 0.51 \\
$\operatorname{Re}=300000$ & 0.47 & 0.46 & 0.45 \\
$\operatorname{Re}=500000$ & 0.43 & 0.42 & 0.42 \\
\hline
\end{tabular}

Reynolds numbers can be highly coincident. When the relative height (ordinate) takes value as 8 , the bottom velocity can reach the mainstream value of the outer layer. According to Table 3, it can be seen that the laminar separation has taken place under all conditions at the position of $x / L=0.4$. Therefore, the viscous sublayer near the wall has begun to appear reverse velocity flow, but with a relatively small value. At the same time, the relative height of the velocity $u$ to the outer velocity $\mathrm{Ue}$ is increased to 10 , and the difference caused by Reynolds number can also be observed. The velocity profiles at $x / L=0.5$ and $x / L=0.6$ have the most obvious differences. Since the maximum momentum thickness of high Reynolds numbers $(R e=300000$ and $R e=500000$ ) has appeared, the velocity profiles begin to fall back. For example, the Re500000 profiles have shown obvious turbulent characteristics, indicating the completion of separation, reattachment, and transition, and the relative height required to reach the outer velocity falls back about to 8 . The relative height of two curves with low Reynolds number $(\operatorname{Re}=70000$ and $R e=150000)$ to reach the outer velocity can reach more than 14, and there is still a distinct reflux velocity and scope. At downstream of $x / L=0.6$, the maximum momentum thickness under the condition of $\mathrm{Re}=$ 150000 has also reached; hence, the velocity profile begins to fall, while $\mathrm{Re}=70000$ still has obvious reflux characteristics, and the relative height is close to 16 . The other two high Reynolds number velocity profiles show more obvious turbulence characteristics. At position $x / L=0.7, \operatorname{Re}=70000$ profile begins to fall, but the attachment is still not completed. Under the other three Reynolds number conditions, the difference between the profiles with obvious turbulence characteristics is getting smaller and smaller; that is to say, they have entered the stage of fully developed turbulence. Overall comparison still shows that the variation of Reynolds number has a stronger effect on the velocity profile than that of turbulence intensity does. With the increase of both Reynolds number and turbulence intensity, a shorter LSB can be obtained, i.e., a faster separation-transition process.

The velocity profile distributions in Figure 12 correspond to the shortest LSB with the Reynolds number equals to 500000. In the figure, only the position $x / L=0.4$ has the velocity distribution of reverse flow, and the difference between the corresponding profiles of different turbulence level is obviously greater than those of other locations. Combining Tables 3 and 4, it can be seen that in the range from $x / L=$ 0.3 to $x / L=0.5$, both flow separation and reattachment are completed, and the range of laminar separation bubbles is obviously smaller than the corresponding values of other conditions. At the downstream of the reattachment position, the turbulent flow profile gradually stabilizes, and the velocity distributions of the last two subfigures are basically the same.

To synthesize $\mathrm{Tu}$ and $\mathrm{Re}$ effects on the time-averaged properties of LSB, the boundary layer integral parameters, such as displacement thickness $\delta^{*}$ and the shape factor $H_{12}$, have been calculated and plotted in Figures 13 and 14. By the distribution of the displacement thickness, the location of the bubble maximum thickness can be quantified, while the peak in the $H_{12}$ distribution is usually related to the transition onset [32-36].

In Figure 13, the simulation results show the effect of Reynolds number on displacement thickness $\delta^{*}$ under the condition of D12-7 and $\mathrm{Tu}=1.5 \%$, with the reference case of $\mathrm{Tu}=5.0 \% \mathrm{Re}=150000$ and two sets of experimental data. The displacement thickness curves corresponding to different Reynolds numbers have the same trend. The trend of $\delta^{*}$ indicates the separation bubble growth, then reduction to reattachment, and reincrease, respectively. Among them, the growth rate of the first stage is greater than that of the third stage, and the thickness corresponding to the location of the reattachment position is also greater than the initial value. When turbulence intensity is fixed, the displacement thickness shrinks evidently and the maximum position moves upstream increasing Reynolds number. This confirms what deduced by the $C_{p}$ curve. The dashed curve in the diagram indicates that turbulence intensity has a weaker influence to LSB's displacement thickness than that of Reynolds number. It also can be seen that the numerical simulation results for laminar separation phenomena have the same trends and characteristic positions according to the experimental ones, especially for the cases under low Reynolds number.

In Figure 14, the LSB's shape factors obtained from simulations are plotted for the same conditions in Figure 12, with the reference case $\mathrm{Tu}=5 \% \mathrm{Re}=150000$ shown with dashed line and two sets of experimental data. It can be seen that the simulation $H_{12}$ distribution curves in low Re cases 

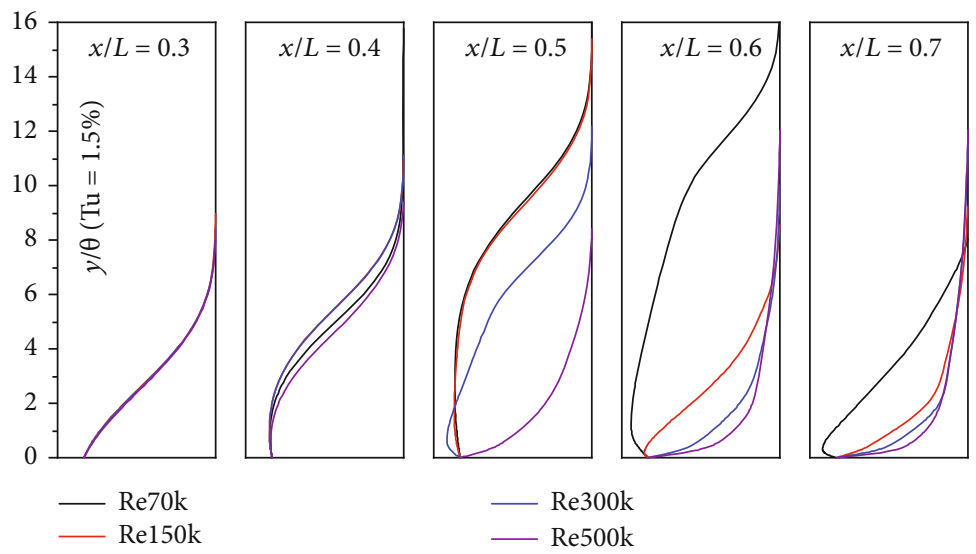

(a)

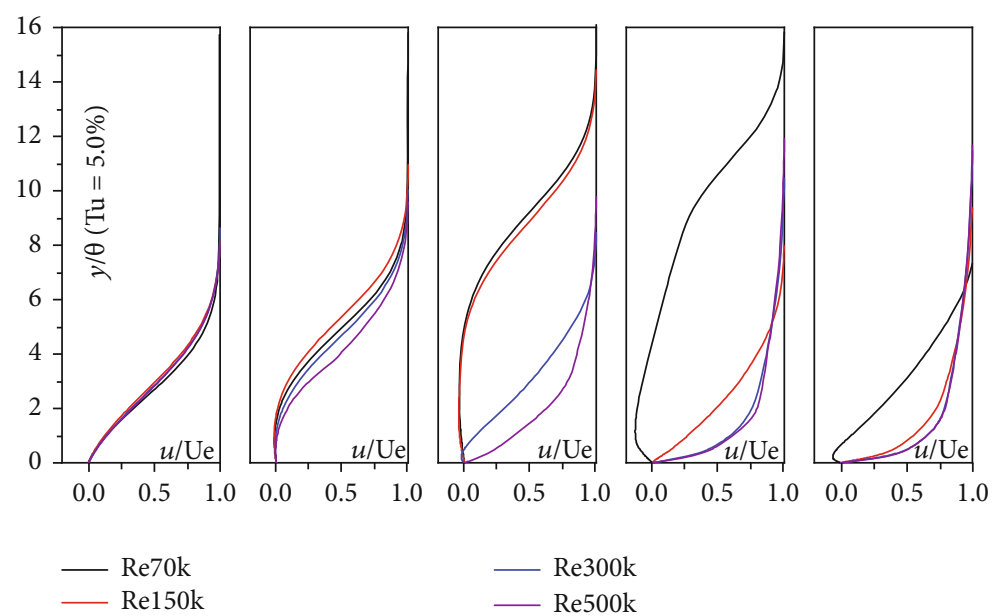

(b)

Figure 11: Velocity profile comparison with fixed $\mathrm{Tu}$. (a) $\mathrm{Tu}=1.5 \%$. (b) $\mathrm{Tu}=5.0 \%$.

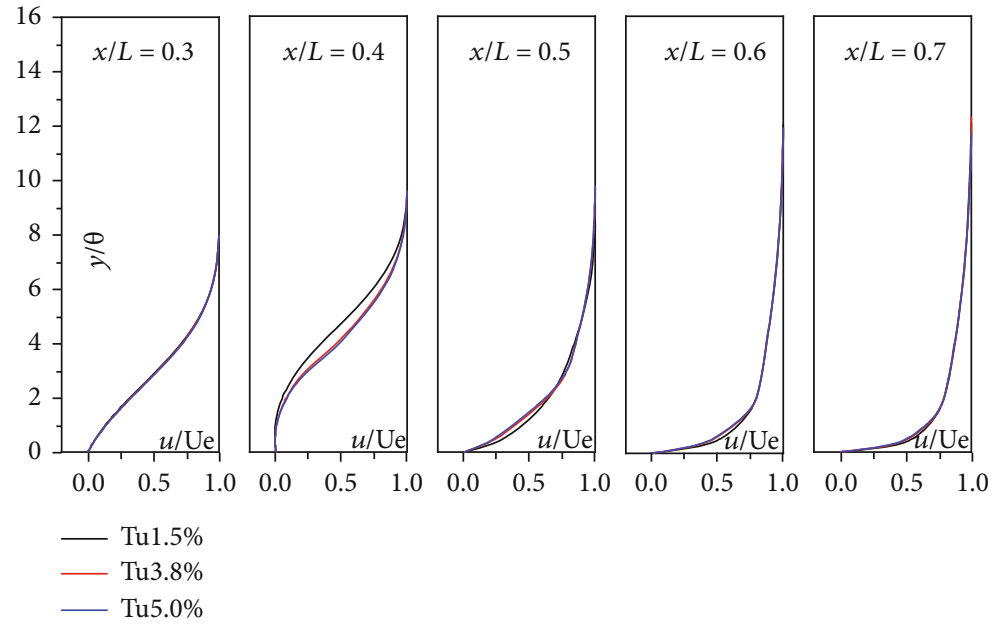

FIgURE 12: Velocity profile comparison with fixed $\mathrm{Re}=500000$.

coincident with experimental ones. From an overall perspective, with fixed turbulence intensity condition, the shape factor distribution reduces increasing the Reynolds number, showing the same trend of the displacement thickness curve.
Moreover, the peak value position of shape factor moves raising the Re number, indicating that the transition point moves forward. The initial shape factor values are equal to 3 in all the cases; then, the curves begin to grow linearly as the 


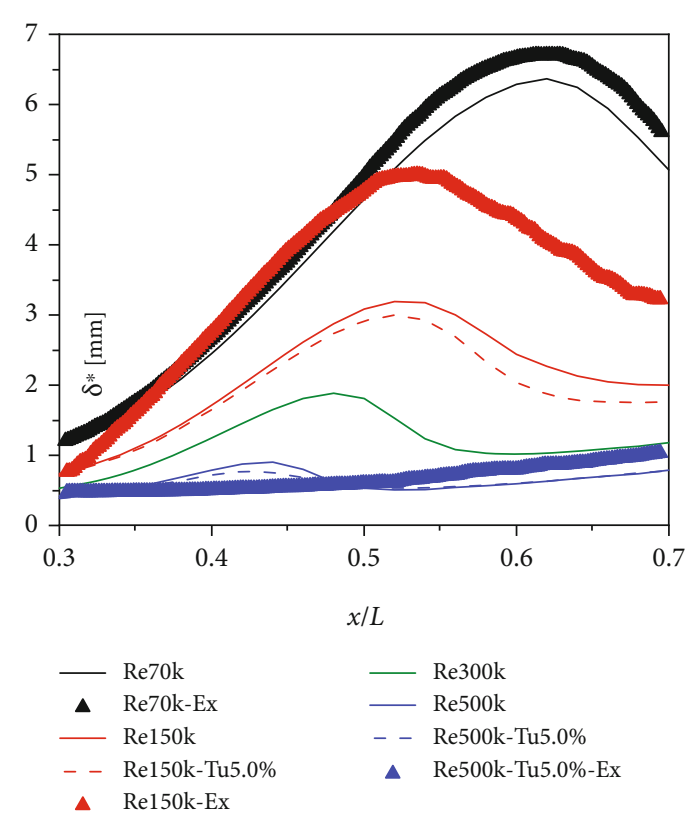

FIgURE 13: Displacement thickness comparison $(\mathrm{Tu}=1.5 \%)$.

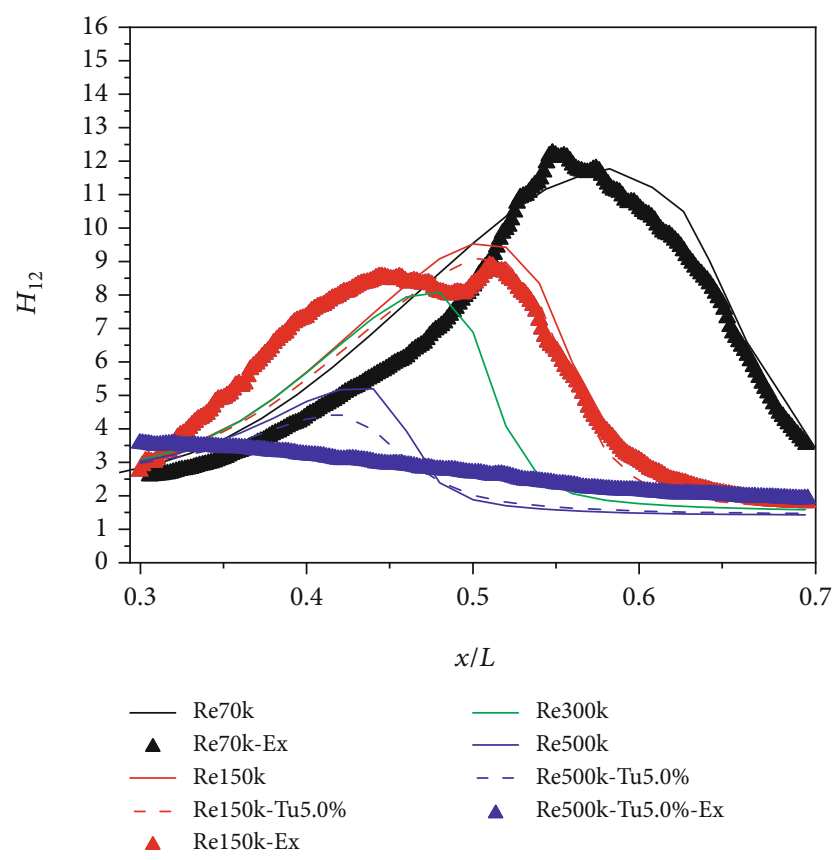

FigURE 14: Shape factor comparison $(\mathrm{Tu}=1.5 \%)$.

separation begins. The position of maximum shape factor indicates the transition onset position. The peak values exceed 4 for every condition, clearly indicating a separated state of the boundary layer. Conversely, at the end of the plate, the curves reduce significantly, showing an asymptotic to a constant value. The shape factor values at the end of the investigated area vary from 1.5 to 1.9 , indicating a fully developed turbulent boundary layer. Comparing the two curves in the cases $\mathrm{Re}=500000$ and $\mathrm{Re}=150000$, it is possible to see that the peak value of the former is about half that of the latter. By the comparison between Figures 13 and 14, it can be
Table 6: Maximum $\delta^{*}$ positions $(x / L)$ with D12-7.

\begin{tabular}{lccc}
\hline & $\mathrm{Tu}=1.5 \%$ & $\mathrm{Tu}=3.8 \%$ & $\mathrm{Tu}=5.0 \%$ \\
\hline $\operatorname{Re}=70000$ & $0.62(0.62-\mathrm{Ex})$ & - & 0.61 \\
$\operatorname{Re}=150000$ & $0.54(0.56-\mathrm{Ex})$ & 0.52 & 0.52 \\
$\operatorname{Re}=300000$ & 0.48 & 0.47 & -0.46 \\
$\operatorname{Re}=500000$ & 0.44 & 0.43 & 0.43 \\
\hline
\end{tabular}

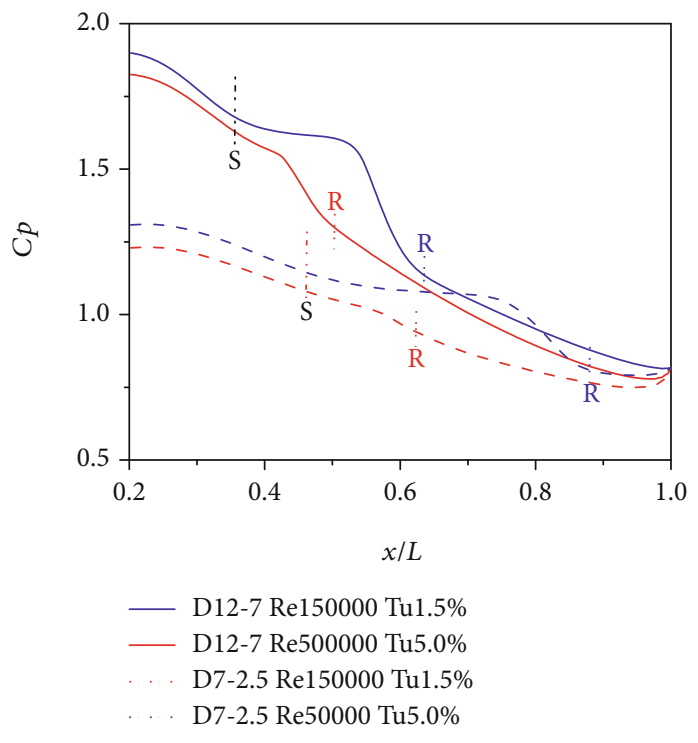

Figure 15: $C_{p}$ comparison with different opening angles.

seen that the maximum displacement thickness position occurs upstream than the transition onset position. The reference case also reveals that the shape factor is more influenced by Reynolds number than the turbulence intensity. To summarize, the maximum displacement thickness and shape factor positions are quantified in Tables 5 and 6. Slight changes are produced when turbulence intensity is changing, similar to Tables 3 and 4.

From Figures 13 and 14, it also can be observed that the laminar displacement thickness and shape factor under high Reynolds number and high turbulence intensity are different from the experiment. There is a bulge in front of the experimental displacement thickness curve distribution, which leads to the difference of the shape factor. The experimental curve shows that in the condition of Re500000-Tu5.0\%, the curve is smooth and without laminar separation zone, which has the similar trends in literature $[15,17]$ at high freestream turbulence level. This means further modification is needed in the transition criterion model in order to well predict bypass transition mechanism.

In the previous analysis, the variation of Reynolds number and turbulence intensity do not have a significant impact on the separation position. In fact, it is affected most by the opening angle of the end-wall. Different end-wall opening angles are adopted during both experiments and numerical simulations; hereby, in Figure 15, the cases corresponding to the shortest and longest LSB are considered. Here, the 
maximum and minimum Reynolds number and turbulence intensity are shown for the opening angle equals to D12-7 and D7-2.5. Data in the figure clearly show that regardless of the end-wall opening angle, the effect of Reynolds number on the separation zone is greater than that of turbulence intensity, in agreement with the previous analysis. Interestingly, reducing the opening angle from D12-7 to D7-2.5, the LSB separating position shifts downstream of the plate, from $x / L=0.36$ to $x / L=0.47$. Also, the reattachment position shifts backwards, and the bubble becomes longer. Indeed, with fixed turbulence intensity and Reynolds number, the distance between detachment and reattachment positions in the case of D7-2.5 is enlarged than case D12-7, which means the LSB's length is stretched by reducing the opening angle.

\section{Conclusions}

Based on the experiments and numerical simulations, a combined investigation about laminar separation bubble on flat plate with adverse pressure gradient is carried out; hence, the effects of Reynolds number, turbulence intensity, and adverse pressure gradient on the overall behavior of laminar separation bubble in time-averaged flow field are obtained. In summary, the variations of Reynolds number and turbulence intensity have marginal effect on the detachment position of LSB, but obviously have influence on the shape and characteristic parameters of LSB, such as bubble extension and maximum thickness. Increasing the opening angle of the end-wall, the detachment position moves upstream, and the length of LSB decreases, which indicates a shorten process of transition from laminar to turbulent. For the condition of fixed APG, Reynolds number and turbulence intensity mainly influence the shape of LSB; Reynolds number provides the greatest influence than turbulent intensity in modifying the LSB. The displacement thickness and shape factor distributions reveal that they are mainly affected by Reynolds number, while the turbulence intensity only induces minor effects. For all conditions, the peak value of shape factor appears earlier than the displacement thickness maximum does, indicating that the transition of LSB begins before the maximum thickness is reached.

The experimental and numerical results are in good agreement with each other, which shows that the simulation scheme with transition criterion $\mathrm{T} \mathrm{k}-\mathrm{kl}-\omega$ model has an acceptable adaptability for predicting the behavior of LSB. Comparatively speaking, the response of the other two turbulence models, T SST and k- $\omega$ SST, to the flat plate LSB is weaker than that of the T k-kl- $\omega$ model. However, the RANS simulation scheme adopted in the paper has an efficient capability in obtaining the time-averaged flow field characteristics of LSB, especially the influence of flow parameters. Thus, this method is of engineering significance for preliminary rapid prediction of the overall behavior of LSB.

\section{Nomenclature}

$C_{p}: \quad$ Pressure coefficient

$D$ : Opening angle degrees of end-wall
$H_{12}$ : Shape factor

L: $\quad$ Length of plate

M: Maximum thickness of boundary layer

R: Reattachment position

Re: Reynolds number

S: Separation position

Tu: Turbulence intensity

$u$ : Velocity component in streamwise

$v$ : Velocity component normal to the plate

$x$ : Streamwise coordinate

$y: \quad$ Normal to the plate coordinate

$y^{+}$: Nondimensional wall coordinate

$\delta^{*}:$ Displacement thickness

$\mu$ : Dynamic viscosity.

\section{Superscripts}

': Fluctuating component

-: Time-averaged value.

\section{Data Availability}

The data used to support the findings of this study are included within the article.

\section{Conflicts of Interest}

The authors declare that there is no conflict of interest regarding the publication of this paper.

\section{Acknowledgments}

This work was supported by the National Natural Science Foundation of China (grant number 51679051), the National Science and Technology Major Project (grant number 2017I-0007-0008), and the Outstanding Youth Foundation of Heilongjiang Province (grant number YQ2019E015). Great acknowledgements are given to the technical support for the experimental portion from Aerodynamics and Turbomachinery Laboratory of the University of Genova.

\section{References}

[1] J. Windte, U. Scholz, and R. Radespiel, "Validierung der RANS-simulation laminarer abloseblasen auf profilen," Aerospace Science and Technology, vol. 10, no. 6, pp. 484-494, 2006.

[2] M. W. Frank, Viscous Flow Fluid, Mc-Graw Hill Company Inc., New York, NY, USA, 3rd edition, 2006.

[3] S. Hermann and G. Klaus, Boundary-Layer Theory, SpringerVerlag, Berlin Heidelberg, Berlin, 9th edition, 2017.

[4] D. Simoni, M. Ubaldi, and P. Zunino, "Experimental investigation of flow instabilities in a laminar separation bubble," Journal of Thermal Science, vol. 23, no. 3, pp. 203-214, 2014.

[5] O. Marxen and D. S. Henningson, "The effect of small amplitude convective disturbances on the size and bursting of a laminar separation bubble," Journal of Fluid Mechanics, vol. 671, pp. 1-33, 2011.

[6] O. Marxen, M. Lang, U. Rist, and S. Wagner, "A combined experimental/numerical study of unsteady phenomena in a laminar separation bubble," Flow, Turbulence and Combustion, vol. 71, no. 1-4, pp. 133-146, 2003. 
[7] C. P. Häggmark, C. Hildings, and D. S. Henningson, “A numerical and experimental study of a transitional separation bubble," Aerospace Science and Technology, vol. 5, no. 5, pp. 317-328, 2001.

[8] S. Yarusevych, J. G. Kawall, and P. E. Sullivan, "Separated shear-layer development on an airfoil at low Reynolds numbers," AIAA Journal, vol. 46, no. 12, pp. 3060-3069, 2008.

[9] A. V. Dovgal, V. V. Kozlov, and A. Michalke, "Laminar boundary layer separation: instability and associated phenomena," Progress in Aerospace Sciences, vol. 30, no. 1, pp. 61-94, 1994.

[10] S. S. Diwan and O. N. Ramesh, "On the origin of the inflectional instability of a laminar separation bubble," Journal of Fluid Mechanics, vol. 629, pp. 263-298, 2009.

[11] U. Maucher, U. Rist, and S. Wagner, "Refined interaction method for direct numerical simulation of transition in separation bubbles," AIAA Journal, vol. 38, no. 8, pp. 1385-1393, 2000.

[12] L. E. Jones, R. D. Sandberg, and N. D. Sandham, "Direct numerical simulations of forced and unforced separation bubbles on an airfoil at incidence," Journal of Fluid Mechanics, vol. 602, pp. 175-207, 2008.

[13] U. Rist, M. Lang, and S. Wagner, "Investigations on controlled transition development in a laminar separation bubble by means of LDA and PIV," Experiments in Fluids, vol. 36, no. 1, pp. 43-52, 2004.

[14] M. Lang, O. Marxen, U. Rist, and S. Wagner, "A Combined Numerical and Experimental Investigation of Transition in a Laminar Separation Bubble," in Recent Results in LaminarTurbulent Transition, pp. 149-164, Springer, 2004.

[15] M. Langari and Z. Yang, "Numerical study of the primary instability in a separated boundary layer transition under elevated free-stream turbulence," Physics of Fluids, vol. 25, no. 7, article 074106, 2013.

[16] H. Li and Z. Yang, "Separated boundary layer transition under pressure gradient in the presence of free-stream turbulence," Physics of Fluids, vol. 31, no. 10, article 104106, 2019.

[17] Z. Yang, "On bypass transition in separation bubbles: a review," Propulsion and Power Research, vol. 8, no. 1, pp. 2334, 2019.

[18] Z. Yang and I. E. Abdalla, "On secondary instability of a transitional separation bubble," Computers \& Fluids, vol. 179, pp. 595-603, 2019.

[19] A. Sengupta and P. Tucker, "Effects of forced frequency oscillations and unsteady wakes on the separation-induced transition in pressure gradient dominated flows," Physics of Fluids, vol. 32, no. 9, article 094113, 2020.

[20] A. Sengupta and P. Tucker, "Effects of forced frequency oscillations and free stream turbulence on the separation-induced transition in pressure gradient dominated flows," Physics of Fluids, vol. 32, no. 10, article 104105, 2020.

[21] R. J. Volino, "Separated flow transition under simulated lowpressure turbine airfoil conditions: part 1 mean flow and turbulence statistics," in ASME Turbo Expo 2002: Power for Land, Sea, and Air, American Society of Mechanical Engineers, pp. 691-702, Journal of Turbomachinery-Transactions of the ASME, 2002.

[22] M. Yaras, "Measurements of the effects of freestream turbulence on separation-bubble transition," in ASME Turbo Expo 2002: Power for Land, Sea, and Air, American Society of Mechanical Engineers, pp. 647-660, Asme Turbo Expo: Power for Land, Sea, \& Air, 2002.
[23] M. Gaster, "The Structure and Behavior of Laminar Separation Bubbles," in AGARD CP4 Part 2, pp. 813-854, Her Majesty's Stationary Office, 1966.

[24] NUMECA, User Manuals, Academic R\&D License, 2019.

[25] A. Fluent, User Manuals, Academic R\&D License, 2019.

[26] D. C. Wilcox, "Reassessment of the scale-determining equation for advanced turbulence models," AIAA Journal, vol. 26, no. 11, pp. 1299-1310, 1988.

[27] D. C. Wilcox, "Comparison of two-equation turbulence models for boundary layers with pressure gradient," AIAA Journal, vol. 31, no. 8, pp. 1414-1421, 1993.

[28] F. R. Menter, "Two-equation eddy-viscosity turbulence models for engineering applications," AIA A Journal, vol. 32, no. 8, pp. 1598-1605, 1994.

[29] F. R. Menter, R. B. Langtry, S. R. Likki, Y. B. Suzen, P. G. Huang, and S. Völker, "A correlation-based transition model using local variables part I: model formulation," Journal of Turbomachinery, vol. 128, no. 3, pp. 413-422, 2006.

[30] R. B. Langtry, F. R. Menter, S. R. Likki, Y. B. Suzen, P. G. Huang, and S. Völker, "A correlation-based transition model using local variables part II: test cases and industrial applications," Journal of Turbomachinery, vol. 128, no. 3, pp. 423434, 2006.

[31] D. K. Walters and D. Cokljat, "A three-equation eddy-viscosity model for Reynolds-Averaged Navier-Stokes simulations of transitional flow," Journal of Fluids Engineering, vol. 130, no. $12,2008$.

[32] R. G. Jacobs and P. A. Durbin, "Simulations of bypass transition," Journal of Fluid Mechanics, vol. 428, pp. 185-212, 2001.

[33] D. Lengani, D. Simoni, M. Ubaldi, P. Zunino, and F. Bertini, "Experimental study of free-stream turbulence induced transition in an adverse pressure gradient," Experimental Thermal and Fluid Science, vol. 84, pp. 18-27, 2017.

[34] D. Simoni, D. Lengani, M. Ubaldi, P. Zunino, and M. Dellacasagrande, "Inspection of the dynamic properties of laminar separation bubbles: free-stream turbulence intensity effects for different Reynolds numbers," Experiments in Fluids, vol. 58 , no. 6, p. 66, 2017.

[35] D. Simoni, M. Ubaldi, P. Zunino, and E. Ampellio, "Freestream turbulence effects on the boundary layer of a high-lift low-pressure-turbine blade," Journal of Thermal Science, vol. 25, no. 3, pp. 195-206, 2016.

[36] D. Simoni, M. Ubaldi, P. Zunino, D. Lengani, and F. Bertini, "An experimental investigation of the separated-flow transition under high-lift turbine blade pressure gradients," Flow, Turbulence and Combustion, vol. 88, no. 1-2, pp. 45-62, 2012. 